\title{
Establishment of a method for calculating standard splenic volume and its use in the evaluation of functional hepatic reserve.
}

\author{
Seika Nakamura, Takeshi Takahara*, Yasushi Hasegawa, Hirokatsu Katagiri, Shouji Kanno, Yuji \\ Akiyama, Takeshi Iwaya, Hiroyuki Nitta, Kouki Otsuka, Keisuke Koeda, Akira Sasaki \\ Department of Surgery, Iwate Medical University School of Medicine, 19-1, Uchimaru, Morioka, Iwate, Japan
}

\begin{abstract}
Background and aim: To objectively assess splenic volume preoperatively, the Standard Splenic Volume (SSV) was calculated for each patient. Whether it could be used to evaluate the functional hepatic reserve less invasively before major hepatectomy was then examined.

Patients and methods: SSV was calculated for 70 patients who had undergone surgery, excluding liver surgery, for benign disease or early-stage cancer. The postoperative clinical courses of 63 patients who had undergone a sectionectomy or hemihepatectomy were then retrospectively investigated by comparing the preoperative splenic volume with each patient's SSV.

Results: The following equation was obtained: SSV $\left(\mathrm{cm}^{3}\right)=177.7 \times$ Body Surface Area $(B S A)-179.9$. None of the patients in the preoperative Splenic Volume (SV)/SSV $<1.1$ group had total Bilirubin (T-Bil) levels $\geq 1.2 \mathrm{mg} / \mathrm{dl}$ in late postoperative period. In contrast, nine $(30 \%)$ of the patients in the preoperative $\mathrm{SV} / \mathrm{SSV} \geq 1.1$ group $(\mathrm{n}=30)$ had T-Bil levels $\geq 1.2 \mathrm{mg} / \mathrm{dl}$ in late postoperative period.

Conclusion: By comparing the preoperative SV and SSV of patients, objective assessments of the patients' preoperative portal venous pressure were obtained noninvasively. We believe that this ratio may provide useful information for evaluating functional hepatic reserve at the time of initial surgery for diseases that may require repeat hepatectomies or multidisciplinary treatment.
\end{abstract}

Keywords: Splenic volume, The standard splenic volume, Functional hepatic reserve.

Accepted on January 30, 2018

\section{Introduction}

The liver and spleen are anatomically connected through the portal venous system, and they are known to be closely related to each other, since both are immunological tissues affected by portal vein pressure. The volumes of the liver and spleen change according to pathological conditions, respectively. Liu et al. reported that the Liver Volume-to-Splenic Volume (LV/SV) ratio decreases with the progression and increasing severity of liver fibrosis in patients with chronic liver diseases [1]. Since the difference in the amount of this decrease between normal individuals and patients with cirrhosis was significant, the authors believe that the LV/SV ratio could serve as an indicator of liver fibrosis. Currently, with the development of various types of simulation software, Liver Volume (LV) and Splenic Volume (SV) can be accurately measured using Computed Tomography (CT) [2,3].

Additionally, Urata et al. investigated 96 normal livers, calculated the body surface area (BSA) from height and weight, measured LV using $\mathrm{CT}$, and derived the following formula for calculating $\mathrm{LV}$ : $\mathrm{LV}(\mathrm{ml})=706.2 \times \mathrm{BSA}\left(\mathrm{m}^{2}\right)+2.4$ $[4,5]$. This is called the Standard Liver Volume (SLV) and is a reliable formula still widely used today, especially as a graft selection criterion for living donor liver transplantation.
However, although SV is measured in several countries, measurement methods vary greatly, and the levels and correlation factors differ greatly among ethnic groups [6-11]. Therefore, a formula for calculating the Standard Splenic Volume (SSV) has yet to be established.

Using CT, along with new simulation software, a formula for calculating the SSV was derived, and whether it could be applied in the clinical setting, such as using it in the evaluation of functional hepatic reserve, was evaluated. The objective of this study was to apply morphometric analyses such as $\mathrm{SV} / \mathrm{SSV}$ to noninvasive evaluations of portal pressure.

\section{Patients and Methods}

This study was conducted after obtaining Institutional Review Board approval from Iwate Medical University School of Medicine. A total of 70 patients ( 23 men, 47 women; mean age $62.4 \mathrm{y}$; age range 50 to $79 \mathrm{y}$ ) who had undergone surgery, excluding liver surgery, for benign and early-stage cancer from December 2007 to November 2013 in our department were analysed (Table 1). All patients were negative for various types of hepatic viruses, did not have hepatic or renal dysfunction, and did not exhibit blood disorders or infections. Four liver transplant donors were also included. 
A volume analyzer, Synapse Vincent version 2 (Fujifilm Medical Company, Tokyo, Japan), was used to measure SV. This instrument allowed automatic rendering of high-precision, three-dimensional (3D) images from CT images. In this study, CT slice thicknesses of 1.0 to $2.5 \mathrm{~mm}$ were used.

$\mathrm{SV}$ was measured from abdominal CT taken within 1 month before surgery. Body Weight (BW) and Height $(\mathrm{BH})$ measurements were obtained at the time of the CT examination or within 1 month before or after the examination, and the Body Mass Index (BMI) and Body Surface Area (BSA) were calculated from these measurements. BMI was calculated using the formula $\mathrm{BMI}=\mathrm{BW} / \mathrm{BH}^{2}$, and $\mathrm{BSA}$ was calculated using the formula BSA $\left(\mathrm{m}^{2}\right)=\mathrm{BW}^{0.425} \times \mathrm{BH}^{0.725} \times 0.007184$ (the DuBois formula) [12]. By examining the correlation between SV and these measurements, we proposed a formula for calculating SSV.

Next, a total of 63 patients who had undergone a sectionectomy or hemihepatectomy in our department were examined for the factors affecting the serum total Bilirubin (TBill) levels at six months after surgery as a late operative setting (Table 2). The Receiver Operating Curves (ROC) were generated for the most affected one among the preoperative factors, and cut off value was set. We divided the 63 patients into two groups. The first group was composed of the 33 patients who had undergone a sectionectomy or hemihepatectomy sometime between 2009 and 2011, while the second group was composed of the 30 patients whose surgeries occurred between 2012 and 2013. In each group, the Receiver Operating Curves (ROC) were generated for the most affected one among the preoperative factors, and, finally, cut-off value was set. The 63 patients were divided into two groups using this cut-off value, and the preoperative factors, intraoperative factors, and postoperative factors were compared between the two groups.

Surgical procedures were classified according to the Brisbane 2000 nomenclature of liver resection.[13] The two groups were then compared for various factors: TBil, Platelet count (Plt), Prothrombin Time (PT) (excluding three people who had taken warfarin orally for Af control purposes), and indocyanine green retention rate at $15 \mathrm{~min}$ (ICG R15) as preoperative factors; intraoperative blood loss volume, transfusion rate, presence/absence of bile leakage (in particular, in cases of insertion of the drain for more than two weeks or a new insertion to drain bile, whether bile leakage had occurred was confirmed), and maximum TBil levels in the immediate postoperative period as intraoperative and early postoperative factors; and the TBil levels at six months after surgery as a late postoperative factor.

\section{Statistical analysis}

The statistical analyses were performed using Stata 13 (Stata Corporation, College Station, TX, USA). In analyses and comparisons of preoperative covariates and clinical parameters, Student's t-test or the Wilcoxon rank-sum test for continuous variables and the $\chi^{2}$ test or Fisher's exact test for categorical variables were used. A p-value $<0.05$ was considered significant. Least-squares regression was used to establish possible linear relationships between the dependent and individual independent variables. The significance of regressions was determined by the F-test. The term $\mathrm{R}^{2}$ was the multiple regression counterpart of $\mathrm{r}^{2}$ and was defined in exactly the same way. As a general rule, corrected $\mathrm{R}^{2}$ was a more useful measure for comparing different regression equations for the same dependent variable. The area under the ROC was calculated to compare the predictive accuracy of the preoperative factor effected on the late postoperative TBil; and the cut-off value of the preoperative factor was calculated by the ROC.

\section{Results}

The mean value of the SV measured using CT in the 70 adult patients was $101.65 \mathrm{ml}$ (range 40.06 to $198.78 \mathrm{ml}$ ). The mean values of each parameter and the correlations between the SV and each parameter are shown in Table 3. Of these parameters, BSA showed the strongest correlation with the SV $(r=0.777$, corrected $\left.\mathrm{R}^{2}=0.598, \mathrm{P}<0.001\right)$. Based on these results, linear regression analysis to predict the SSV was performed, and the following equation was obtained: $\operatorname{SSV}\left(\mathrm{cm}^{3}\right)=177.7 \times$ BSA-179.9. In addition, the correlation between the SV and BSA was as shown in Figure 1.

\section{$\underline{S S V}=177.7 \times$ BSA -179.9}

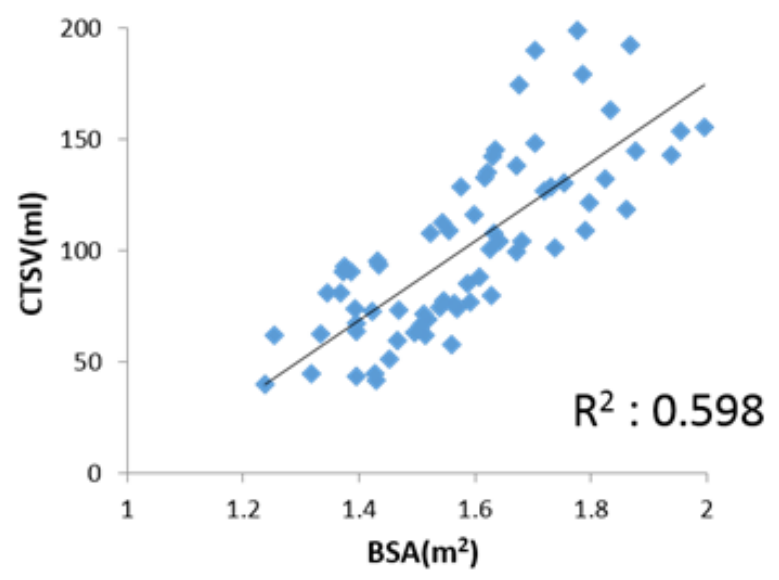

Figure 1. The equation of SSV.

An odds ratio was taken to examine the association between late postoperative TBil and preoperative PT, ICG R15, SV/ SSV, intraoperative blood loss, and hepatic resection rate in 63 patients after sectionectomy or hemihepatectomy. The strongest correlative factor was SV/SSV (Table 4). Furthermore, creating the ROC for SV/SSV, the cut off value became 1.1. Furthermore, the area under ROC for SV/SSV in the first 33 patients was 0.75 , and the area under ROC in the second 30 patients was 0.816 . For this reason, the cut-off value of SV/SSV became 1.1. 
Establishment of a method for calculating standard splenic volume and its use in the evaluation of functional hepatic reserve

A total of 63 patients were divided into two groups: those with a preoperative $\mathrm{SV} / \mathrm{SSV}<1.1$ (Group $\mathrm{A}$ ), and those with a preoperative SV/SSV $\geq 1.1$ (Group B).

The groups were then compared for preoperative, intraoperative and postoperative factors. Although there were significant cases of hemihepatectomy in Group B, no significant difference was observed in the ratio of remnant $\mathrm{LV}$ to total LV.

Preoperative and postoperative albumin (Alb), Plt, PT, TBil, and intraoperative blood loss volume were examined between the two groups, and preoperative Alb value and intraoperative blood loss volume were significant different in group B (Table 5). With respect to the patients' postoperative TBil levels at six months after surgery, none of the 33 patients in group A had six-month postoperative TBil levels $\geq 1.2 \mathrm{mg} / \mathrm{dl}$. In contrast, nine of the 30 patients in Group B had six-month postoperative TBil levels $\geq 1.2 \mathrm{mg} / \mathrm{dl}$.

Table 1. Characteristics of the 70 enrolled patients to calculate the SV.

\begin{tabular}{ll}
\hline Diagnosis & $\mathbf{n}$ \\
\hline Breast cancer & 28 \\
\hline Gastric cancer & 12 \\
\hline Colon cancer & 11 \\
\hline Rectal cancer & 2 \\
\hline Gallstone & 12 \\
\hline Gastrointestinal stromal tumor & 1 \\
\hline Living liver donor & 4 \\
\hline
\end{tabular}

Table 2. Characteristics of the 63 patients who underwent sectionectomy or hemihepatectomy.

\begin{tabular}{ll}
\hline Analysed factor & $\mathbf{n}$ \\
\hline Diagnosis & \\
\hline Hepatocellular carcinoma & 28 \\
\hline Liver metastasis & 26 \\
\hline Cholangiocellular carcinoma & 5 \\
\hline
\end{tabular}

\begin{tabular}{ll}
\hline Intrahepatic cholelithiasis & 2 \\
\hline Others & 2 \\
\hline Operative procedure & 8 \\
\hline Left lateral sectionectomy & 7 \\
\hline Anterior sectionectomy & 12 \\
\hline Posterior sectionectomy & 4 \\
\hline Central sectionectomy & 32 \\
\hline Heminepatectomy & \\
\hline Hepatic virus infection & 10 \\
\hline HBV & 7 \\
\hline HCV & \\
\hline
\end{tabular}

Table 3. Parameters of the 70 enrolled patients and correlations between $S V$ and these parameters.

\begin{tabular}{lllll}
\hline Analysed factor & Mean \pm SD & Range & $\mathbf{R}$ & Corrected $\mathbf{R}^{2}$ \\
\hline $\mathrm{SV}(\mathrm{ml})$ & $101.65 \pm 39.68$ & $40.06-198.78$ & - & - \\
\hline $\mathrm{BH}(\mathrm{cm})$ & $158.30 \pm 9.32$ & $135.9-180$ & 0.631 & 0.389 \\
\hline $\mathrm{BW}(\mathrm{kg})$ & $58.11 \pm 10.50$ & $37.6-88$ & 0.762 & 0.575 \\
\hline $\mathrm{BMl}\left(\mathrm{kg} / \mathrm{m}^{2}\right)$ & $23.06 \pm 3.00$ & $17.2-30.8$ & 0.472 & 0.211 \\
\hline $\mathrm{BSA}\left(\mathrm{m}^{2}\right)$ & $1.59 \pm 0.17$ & $1.239-1.996$ & 0.777 & 0.598 \\
\hline Age $(\mathrm{y})$ & $62.16 \pm 6.91$ & $50-79$ & 0.009 & -0.015 \\
\hline
\end{tabular}

Table 4. Preoperative factors influencing postoperative T-Bil levels at 6 months after major hepatectomy.

\begin{tabular}{lllll}
\hline Analysed factor & Odds ratio & Std. Err. & $\mathbf{p}$ & $\mathbf{9 5 \%} \mathbf{C l}$ \\
\hline SVISSV & 1.829 & 0.761 & 0.147 & $0.809-4.134$ \\
\hline Resection rate & 1.033 & 0.027 & 0.216 & $0.982-1.086$ \\
\hline ICG R15 & 1.057 & 0.088 & 0.508 & $0.897-1.245$ \\
\hline Blood loss & 1 & 0.001 & 0.997 & $0.999-1.001$ \\
\hline PT & 1.188 & 0.415 & 0.622 & $0.599-2.355$ \\
\hline
\end{tabular}

Table 5. Parameters of groups $A$ and $B$.

\begin{tabular}{lllll}
\hline Analysed factor & Group A (n=33) & Group B (n=30) & $\mathbf{p}$ & 0.959 \\
\hline Age $(\mathrm{y})$ & & $64.4 \pm 7.0$ & $64.3 \pm 7.8$ & $187.7 \pm 64.1$ \\
\hline $\mathrm{SV}(\mathrm{ml})$ & & $91.7 \pm 28.7$ & $113.7 \pm 34.6$ & 0.6 .001 \\
\hline $\mathrm{SSV}(\mathrm{ml})$ & $110.1 \pm 23.7$ & $10.5 \pm 4.7$ & 0.991 \\
\hline ICG R15 & & $10.5 \pm 5.7$ & 2 & 0.261 \\
\hline Operative procedure & Left lateral sectionectomy & 6 & 3 & 1 \\
\cline { 2 - 5 } & Anterior sectionectomy & 4 & 3 & 0.112 \\
\cline { 2 - 5 } & Posterior sectionectomy & 9 & & \\
\hline
\end{tabular}




\begin{tabular}{|c|c|c|c|}
\hline Central sectionectomy & 4 & 0 & 0.115 \\
\hline Hemihepatectomy & 10 & 22 & $<0.001$ \\
\hline Preoperative Alb (g/dl) & $4.16 \pm 0.42$ & $3.78 \pm 0.61$ & 0.005 \\
\hline Preoperative PIt $\left(10^{4} \mu \mathrm{l}\right)$ & $19.7 \pm 9.4$ & $20.8 \pm 8.8$ & 0.408 \\
\hline Preoperative PT (s) & $11.2 \pm 0.01$ & $11.66 \pm 1.93$ & 0.099 \\
\hline Preoperative TBil (mg/dl) & $0.55 \pm 0.21$ & $0.64 \pm 0.39$ & 0.26 \\
\hline Preoperative TBil>1.2, n (\%) & $0(0 \%)$ & $2(6.7 \%)$ & 0.223 \\
\hline Intraoperative blood loss (g) & $277.5 \pm 224.1$ & $674.4 \pm 1020.7$ & 0.046 \\
\hline Intra or postoperative transfusion, $\mathrm{n}(\%)$ & $4(12.1 \%)$ & $6(20.0 \%)$ & 0.498 \\
\hline Secondary bleeding, n (\%) & $2(6.1 \%)$ & $2(6.7 \%)$ & 1 \\
\hline Bile leakage, n (\%) & $7(21.2 \%)$ & $3(10 \%)$ & 0.308 \\
\hline Postoperative Alb (g/dl) & $3.74 \pm 0.43$ & $3.70 \pm 0.51$ & 0.129 \\
\hline Postoperative PIt $\left(10^{4} \mu \mathrm{l}\right)$ & $20.8 \pm 7.8$ & $19.4 \pm 8.7$ & 0.529 \\
\hline Postoperative PT (s) & $11.6 \pm 0.19$ & $12.1 \pm 0.3$ & 0.21 \\
\hline Postoperative Max Tbil (mg/dl) & $1.95 \pm 1.07$ & $2.4 \pm 1.61$ & 0.185 \\
\hline Postoperative Tbil at 3 months (mg/dl) & $0.58 \pm 0.24$ & $0.79 \pm 0.91$ & 0.241 \\
\hline Postoperative Tbil at 6 months>1.2, $\mathrm{n}(\%)$ & $0(0 \%)$ & $9(30 \%)$ & $<0.001$ \\
\hline The ratio of remnant liver volume of Hemihepatectomy (\%) & $54.59 \pm 11.01$ & $64.08 \pm 16.15$ & 0.164 \\
\hline The ratio of remnant liver volume of hepatectomy (\%) & $69.30 \pm 16.05$ & $61.61 \pm 15.38$ & 0.069 \\
\hline
\end{tabular}

\section{Discussion}

Portal venous pressure is one of important factors in the preoperative evaluation of functional hepatic reserve. In addition, portal hypertension and splenomegaly are known to correlate with each other. Two mechanisms are responsible for the splenomegaly secondary to portal hypertension $[14,15]$.

Hayashi et al. measured portal venous pressure in 40 patients with liver tumors who required portal vein puncture for conducting multiple regression analyses on the association between portal venous pressure and noninvasive parameters [16]. The authors found that, among the parameters, TBil and SV were positively associated with portal venous pressure, and that an elevated TBil and SV were particularly strong predictors of early and advanced portal hypertension, respectively. The study indicated that portal venous pressure can be noninvasively monitored by concomitantly measuring both parameters. However, it is difficult to noninvasively evaluate portal venous pressure and assess the preoperative liver reserve at the same time in each patient. Hepatic functional reserve is often affected by portal tension, and on the other hand, portal hyper-tension leads to splenomegaly. We presumed that we can evaluate subclinical portal-hyper tension by calculating the SV/SSV, so, the SV/SSV of each patient might be one of the measurements for hepatic functional reserve.

Heymsfield et al. used CT to measure splenic mass, calculating it by totalling the areas of each slice [17]. The calculated value showed a significant correlation with the splenic mass determined at autopsy. Kaneko et al. measured spleen volume in 150 healthy adults who later became liver transplant donors and reported that their mean spleen volume was $112 \mathrm{~cm}^{3}$ [18]. In the present study, Fujifilm Medical Company's Synapse Vincent version 2, a 3D image analysis system that transforms two-dimensional $\mathrm{CT}$ images to 3D images, was used to automatically measure SV. In the present study, the mean spleen weight was $101.65 \pm 39.68 \mathrm{ml}$, a value slightly lower than that reported by Kaneko et al. In the present study, patients who had undergone a sectionectomy or hemihepatectomy, the majority of whom were 50-79 y old, were investigated. In general, the spleen decreases in size with age after peaking during early adulthood. Limiting the study subjects to those who were 50-79 y old may have been the reason for the lower observed spleen weight in this study compared to the values reported by Kaneko et al. Furthermore, when study subjects were limited to this age group and the age parameter was excluded, BSA was the parameter with the strongest correlation with spleen weight. There are several limitations in this study. Spleen volume might be affected by a patient's age, so we limited the participants to those patients between the ages of 50 and $79 \mathrm{y}$ old. We chose this age range because most of the patients who underwent sectionectomies and hemihepatectomies were 50-79 y old. Another limitation is that we recruited patients with early-stage cancer to calculate a formula of SSV, because there were few opportunities to do this when a normal, healthy subject received a CT. 
In the present study, a formula for calculating the SSV was determined using CT in 63 patients who underwent a sectionectomy or hemihepatectomy, and the clinical applicability of this formula was evaluated by assessing its relationship with the preoperative SV. Although none of the 33 patients $(0 \%)$ in group $\mathrm{A}$ had postoperative TBil levels $\geq 1.2$ $\mathrm{mg} / \mathrm{dl}$, nine of the 30 patients $(30 \%)$ in group B had postoperative TBil levels $\geq 1.2 \mathrm{mg} / \mathrm{dl}$.

As shown in the results in Table 5, there are many cases of hemihepatectomy in group B, it was considered that there is a possibility that postoperative TBil levels may be prolonged as the operation procedure for an extensive hepatectomy is performed. However, as there was no significant difference in the ratio of remnant LV to total LV between the two groups, it is unlikely that the operative procedure is a factor influencing the postoperative liver function.

As shown in Table 5, there were no significant differences between groups A and B in the preoperative ICG R15 value, preoperative TBil levels, including the platelet count. However, the two groups showed a significant difference in preoperative Alb value and intraoperative blood loss. Although there was a significant difference in preoperative Alb value, both groups had normal values (Alb: $\geq 3.7 \mathrm{~g} / \mathrm{dL}$ ), suggesting that it is unlikely to be a factor that prolongs postoperative liver damage.

We surmised that this was because the patient groups had similar reserve functions in the background liver and had normal bilirubin production, metabolism, and excretion. For the 30 patients in group B, the amount of blood loss in the nine patients with a postoperative TBil levels $\geq 1.2 \mathrm{mg} / \mathrm{dl}$ was not significantly different from that in the other 21 patients, while a comparison of blood loss between those nine patients and the 33 patients in Group A also showed no significant difference. Based on these findings, we concluded that intraoperative blood loss is not necessarily related to elevated postoperative TBil levels.

Preoperative TBil levels were above the normal limit $(\geq 1.2$ $\mathrm{mg} / \mathrm{dl}$ ) in two of the patients in Group B, with the high TBil value persisting postoperatively in one of them. That patient was suspected of having Gilbert's syndrome and, thus, had elevated T-Bil throughout the perioperative period. In addition, eight patients had higher-than-normal postoperative TBil levels despite having normal preoperative TBil levels. None of these eight patients had received a transfusion, and the preoperative ICG R15 was also $\leq 19 \%$ in each. In addition, in the background liver, fatty liver and chronic hepatitis were seen in about half of the patients, while liver cirrhosis was seen in only one. These eight patients may have had latent portal hypertension, although this could not be inferred from the preoperative blood and biochemical findings.

Clear evidence of portal hypertension that accompanies primary liver diseases, such as tumors or liver fibrosis, was not observed in group A, which suggested that liver regeneration after hepatectomy would occur normally in these patients. This suggests that postoperative functional hepatic reserve is sufficiently secured as long as the preoperative CTSV/SSV is $<1.1$, even in cases that require a sectionectomy or hemihepatectomy. However, this also suggests that, in patients whose preoperative CTSV/SSV is $\geq 1.1$, a sectionectomy or hemihepatectomy may worsen their postoperative functional hepatic reserve compared to their preoperative levels despite an uneventful perioperative course.

\section{Conclusion}

In the present study, preoperative SV and SSV were compared in patients who underwent a sectionectomy or hemihepatectomy, and an objective measure of preoperative splenomegaly and, thus, portal venous pressure was obtained. In particular, the present results suggest that prolonged postoperative TBil levels $\geq 1.2 \mathrm{mg} / \mathrm{dl}$ may occur when the ratio of preoperative $\mathrm{SV} / \mathrm{SSV}$ is $\geq 1.1$. In diseases that may require repeat hepatectomies or multidisciplinary treatment, such as colorectal liver metastasis or hepatocellular carcinoma, this ratio of preoperative $\mathrm{SV}$ to the SSV may provide useful information for evaluating the functional hepatic reserve at the time of initial surgery.

\section{Ethics Approval and Consent to Participate}

This study was conducted after obtaining Institutional Review Board approval from Iwate Medical University School of Medicine.

\section{Consent for Publication}

Written informed consent was obtained from the patient for publication of this study.

\section{Availability of Data and Materials}

Not applicable

\section{Competing Interests}

The authors declare no conflicts of interest.

\section{Funding}

None

\section{Authors' Contributions}

$\mathrm{SN}$ is the main author of this article. SN, TT and YH conceived this study. TT, YH and AS supervised the manuscript writing. $\mathrm{SN}, \mathrm{HK}, \mathrm{SK}, \mathrm{YA}, \mathrm{TI}, \mathrm{HN}, \mathrm{KO}$ and $\mathrm{KK}$ contributed to the collection of clinical information and data analysis. TT and $\mathrm{YH}$ reviewed the manuscript and revised it thoroughly. All authors have read and approved the final manuscript.

\section{Acknowledgements}

Not applicable 


\section{References}

1. Liu P, Li P, He W, Zhao LQ. Liver and spleen volume variations in patients with hepatic fibrosis. World J Gastroenterol 2009; 15: 3298-3302.

2. Takamoto T, Hashimoto T, Ogata S, Inoue K, Maruyama Y, Miyazaki A, Makuuchi M. Planning of anatomical liver segmentectomy and subsegmentectomy with 3-dimensional simulation software. Am J Surg 2013; 206: 530-538.

3. Smith AD, Branch CR, Zand K, Subramony C, Zhang H, Thaggard K, Hosch R, Bryan J, Vasanji A, Griswold M, Zhang $\mathrm{X}$. Liver surface nodularity quantification from routine CT images as a biomarker for detection and evaluation of cirrhosis. Radiology 2016; 151542.

4. Urata K, Kawasaki S, Matsunami H, Hashikura Y, Ikegami T, Ishizone S, Momose Y, Komiyama A, Makuuchi M. Calculation of child and adult standard liver volume for liver transplantation. Hepatology 1995; 21: 1317-1321.

5. Um EH, Hwang S, Song GW, Jung DH, Ahn CS, Kim KH, Moon DB, Park GC, Lee SG. Calculation of standard liver volume in Korean adults with analysis of confounding variables. Korean J Hepatobiliary Pancreat Surg 2015; 19: 133-138.

6. Hidaka H, Nakazawa T, Wang G, Kokubu S, Minamino T, Takada J, Tanaka Y, Okuwaki Y, Watanabe M, Shibuya A, Koizumi W. Reliability and validity of splenic volume measurement by 3-D ultrasound. Hepatol Res 2010; 40: 979-988.

7. Picardi M, Martinelli V, Ciancia R, Soscia E, Morante R, Sodano A, Fortunato G, Rotoli B. Measurement of spleen volume by ultrasound scanning in patients with thrombocytosis: a prospective study. Blood 2002; 99: 4228-4230.

8. Prassopoulos P, Daskalogiannaki M, Raissaki M, Hatjidakis A, Gourtsoyiannis N. Determination of normal splenic volume on computed tomography in relation to age, gender and body habitus. Eur Radiol 1997; 7: 246-248.

9. Hoefs JC, Wang FW, Lilien DL, Walker B, Kanel G. A novel, simple method of functional spleen volume calculation by liver-spleen scan. J Nucl Med 1999; 40: 1745-1755.

10. Asghar A, Agrawal D, Yunus SM, Sharma PK, Zaidi SH, Sinha A. Standard splenic volume estimation in north indian adult population: using 3D reconstruction of abdominal CT scan images. Anat Res Int 2011; 2011: 707325.

11. Mazonakis M, Damilakis J, Maris T, Prassopoulos P, Gourtsoyiannis N. Estimation of spleen volume using MR imaging and a random marking technique. Eur Radiol 2000; 10: 1899-1903.

12. Du Bois D, Du Bois EF. A formula to estimate the approximate surface area if height and weight be known. Nutrition 1989; 5: 303-311.

13. Strasberg SM. Nomenclature of hepatic anatomy and resections: a review of the Brisbane 2000 system. J Hepatobiliary Pancreat Surg 2005; 12: 351-355.

14. Mejias M, Garcia-Pras E, Gallego J, Mendez R, Bosch J, Fernandez M. Relevance of the mTOR signaling pathway in the pathophysiology of splenomegaly in rats with chronic portal hypertension. J Hepatol 2010; 52: 529-539.

15. Li ZF, Zhang S, Huang Y, Xia XM, Li AM, Pan D, Zhang W, Wang J. Morphological changes of blood spleen barrier in portal hypertensive spleen. Chin Med J (Engl) 2008; 121: 561-565.

16. Hayashi H, Beppu T, Okabe H, Nitta H, Imai K, Doi K, Chikamoto A, Baba H. Combined measurements of serum bile acid level and splenic volume may be useful to noninvasively assess portal venous pressure. J Gastroenterol 2012; 47: 1336-1341.

17. Heymsfield SB, Fulenwider T, Nordlinger B, Barlow R, Sones P, Kutner M. Accurate measurement of liver, kidney, and spleen volume and mass by computerized axial tomography. Ann Intern Med 1979; 90: 185-187.

18. Kaneko J, Sugawara Y, Matsui Y, Ohkubo T, Makuuchi M. Normal splenic volume in adults by computed tomography. Hepatogastroenterology 2002; 49: 1726-1727.

\section{*Correspondence to}

Takeshi Takahara

Department of Surgery

Iwate Medical University School of Medicine

Japan 